\title{
Introduction to the CPAIOR 2020 fast track issue
}

\author{
Emmanuel Hebrard ${ }^{1} \cdot$ Nysret Musliu ${ }^{2}$
}

Accepted: 12 November 2020Published online: 08 December 2020

(C) The Author(s), under exclusive licence to Springer Science+Business Media, LLC part of Springer Nature 2021

The 17th International Conference on the Integration of Constraint Programming, Artificial Intelligence, and Operations Research (CPAIOR 2020), exceptionally held online from Vienna, Austria, September 21-24, 2020.

The aim of the CPAIOR conference is to bring together interested researchers from Constraint Programming (CP), Artificial Intelligence (AI), and Operations Research (OR) to present new techniques or applications, and to provide an opportunity for researchers in one area to learn about techniques in the others. A main objective of this conference series is also to give these researchers the opportunity to show how the integration of techniques from different fields can lead to interesting results on large and complex problems.

All papers submitted to the conference were subject to a rigorous peer-review process. Accepted papers were published in the conference proceedings [1], except for a small selection of outstanding papers, invited to be published exclusively through a fast-track process in the Constraints journal. Before being accepted into Constraints, these papers were subject to another round of reviews and revisions to ensure the high standards for this journal were met.

The four following papers accepted to the fast track give an excellent snapshot of the papers accepted at CPAIOR 2020, especially the core aspect of the conference: the hybridization of Operations Research and Artificial Intelligence techniques, and their application to diverse domains:

The paper Power of Pre-Processing: Production Scheduling with Variable Energy Pricing and Power-Saving States by Ondřej Benedikt, István Módos and Zdeněk Hanzálek investigates a single-machine scheduling problem with variable energy costs and powersaving machine states. A novel pre-processing technique is proposed to compute the optimal

This article belongs to the Topical Collection: Special Issue on Constraint Programming, Artificial Intelligence, and Operations Research

Guest Editors: Emmanuel Hebrard and Nysret Musliu

Emmanuel Hebrard

hebrard@laas.fr

Nysret Musliu

musliu@dbai.tuwien.ac.at

2 Christian Doppler Laboratory for Artificial Intelligence and Optimization for Planning and Scheduling, DBAI, TU Wien, Vienna, Austria 
switching of the machine states with respect to the energy costs. To solve the problem, integer linear programming and constraint programming models are proposed and evaluated on a set of benchmark instances.

The paper The Potential of Quantum Annealing for Rapid Solution Structure Identification by Yuchen Pang, Carleton Coffrin, Andrey Y. Lokhov and Marc Vuffray, thoroughly benchmarks Quantum Annealers against several standard optimization approaches on a class of combinatorial problems on Ising models. The key insight of this study is that there actually are classes of problems where Quantum Annealers converge quickly toward the global optimum, whereas classic algorithms are stuck on - or take significantly more time to escape - local optima.

The paper A New Constraint Programming Model and Solving for the Cyclic Hoist Scheduling Problem by Mark Wallace Neil Yorke-Smith studies an important practical problem. Authors propose a new constraint programming model that is simpler, yet flexible enough to accommodate different variants of cyclic hoist scheduling problem. To solve the model various approaches are applied including lazy clause generation. The paper also introduces a robust parallel hybrid approach that combines integer programming and lazy clause generation.

The paper Learn to Relax: Integrating 0-1 Integer Linear Programming with PseudoBoolean Conflict-Driven Search by Jo Devriendt Ambros Gleixner, and Jakob Nordström, to be published in a future issue, proposes an innovative approach to integrating an LP solver and cut generation into a pseudo-Boolean solver. The main challenge is that solving the LP relaxation after each decision defeats an essential feature of pseudo-Boolean solvers: the speed of the reasoning step. This issue is tackled by carefully limiting the computation time when interleaving incremental LP relaxations within conflict-driven search.

We would like to thank the Program Committee members for the initial reviews and for helping us selecting the most relevant papers, and we would also like to thank the additional reviewers for their help with the second round of peer-reviewing for the Constraints journal. Of course, we also thank the authors for submitting high-quality papers and for revising and extending them to the articles published in this issue.

\section{References}

1. Hebrard, E., \& Musliu, N. (eds.). (2020). Integration of constraint programming, artificial intelligence, and operations research - 17th international conference, CPAIOR 2020, Vienna, Austria, September 2124, 2020, proceedings, volume 12296 of lecture notes in computer science. Springer.

Publisher's note Springer Nature remains neutral with regard to jurisdictional claims in published maps and institutional affiliations. 